\title{
Mn(III) Chain Coordination Polymers assembled by Salicylidene-2- ethanolamine Schiff Base Ligands: Synthesis, Crystal Structures and HFEPR Study
}

Oleh Stetsiuk ${ }^{\mathrm{a}, \mathrm{c}}$, Nataliya Plyuta ${ }^{\mathrm{a}, \mathrm{c}}$, Narcis Avarvari ${ }^{\mathrm{a}}$,Evgeny Goreshnik ${ }^{\mathrm{b}}$, Vladimir Kokozay, Svitlana Petrusenko ${ }^{*}$, and Andrew Ozarowski ${ }^{* d}$

${ }^{a}$ MOLTECH-Anjou, UMR 6200, CNRS, UNIV Angers, 2 bd Lavoisier, 49045 ANGERS Cedex, France

${ }^{b}$ Department of Inorganic Chemistry and Technology, Jožef Stefan Institute, Jamova 39, 1000 Ljubljana, Slovenia

${ }^{c}$ Department of Inorganic Chemistry, Taras Shevchenko National University of Kyiv, Volodymyrska str.64/13, Kyiv01601,Ukraine.E-mail: spetrusenko@yahoo.com ${ }^{d}$ National High Magnetic Field Laboratory, Florida State University, 1800 E. Paul Dirac Drive, Tallahassee, Florida 32310,USA.E-mail: ozarowsk@magnet.fsu.edu; Fax: +1850$644-1366$

\section{Supplementary information}




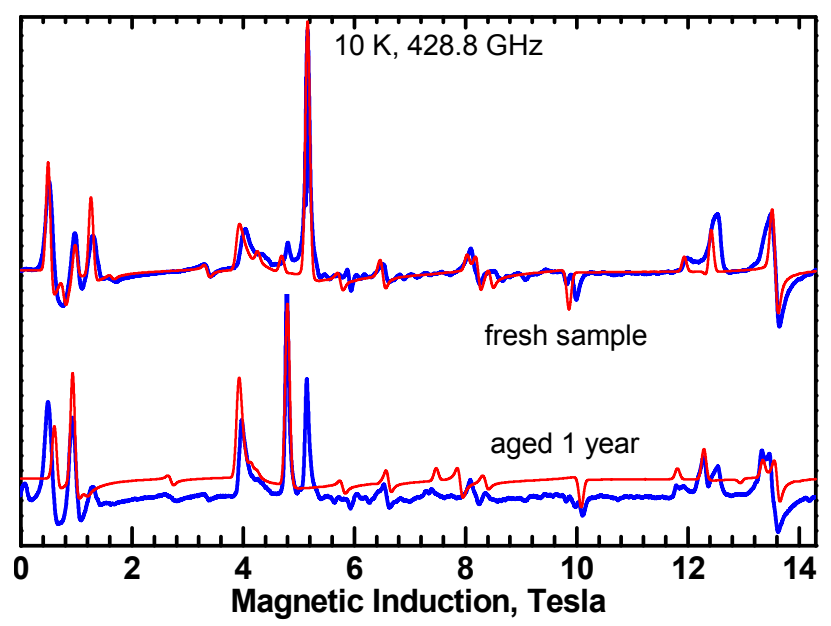

Figure S1. EPR spectra of 1. Blue: experimental; red: simulated. The spin Hamiltonian parameters of the fresh sample are given in Table 4 (main text). The spectrum of an aged sample was simulated using $\mathrm{g}_{\mathrm{x}}=1.976, \mathrm{~g}_{\mathrm{y}}=$ 1.976, $\mathrm{g}_{\mathrm{z}}=2.002, \mathrm{D}=-3.37 \mathrm{~cm}^{-1}, \mathrm{E}=-0.68 \mathrm{~cm}^{-1}, \mathrm{~B}_{4}{ }^{0}=-0.00051 \mathrm{~cm}^{-1}, \mathrm{~B}_{4}{ }^{4}=-0.0073 \mathrm{~cm}^{-1}$.

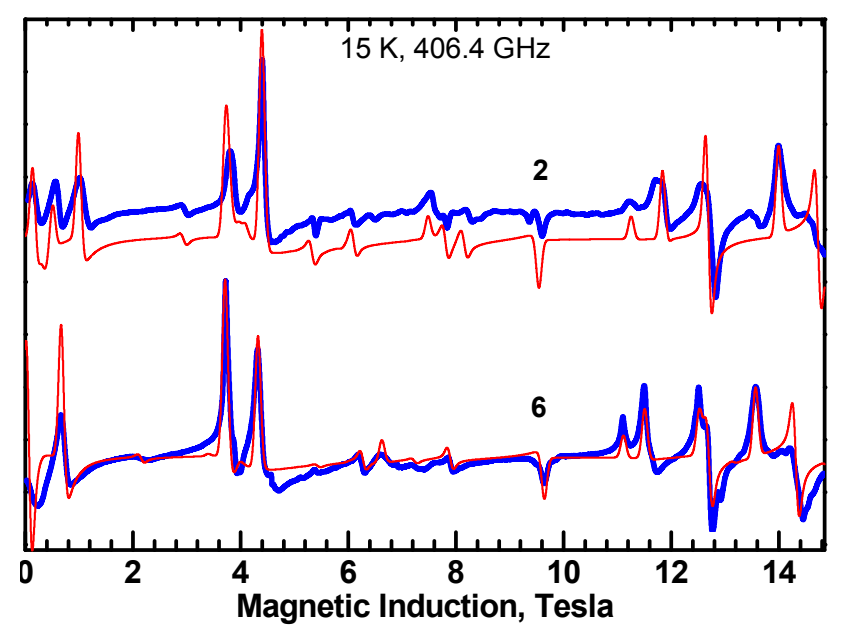

Figure S2. EPR spectra of the $\mathrm{Br}^{-}$- containing complexes 2 (L1) and 6 (L2). Blue: experimental; red: simulated. The spin Hamiltonian parameters are given in Table 4 (main text). 


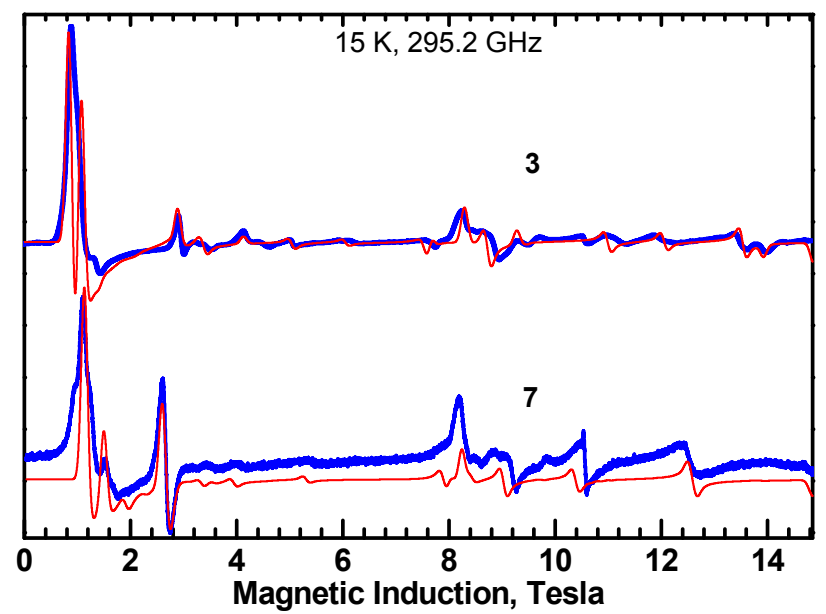

Figure S3. EPR spectra of the $\mathrm{I}^{-}$- containing complexes 3 (L1) and 7 (L2). Blue: experimental; red: simulated. The spin Hamiltonian parameters are given in Table 4 (main text).

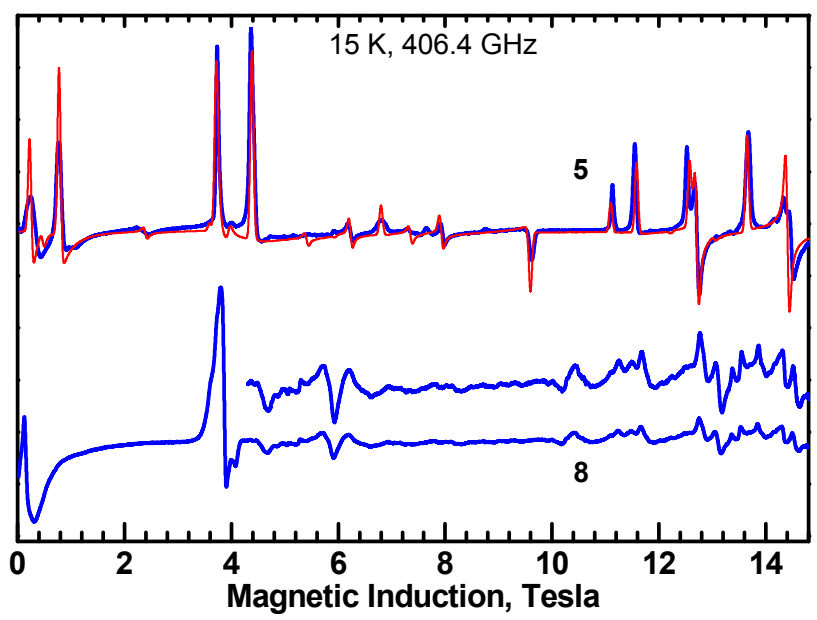

Figure S4. Comparison of the EPR spectra of the well-behaved complex 5 to ill-behaved 8. Blue: experimental; red: simulated. The spin Hamiltonian parameters are given in Table 4 (main text). The additional splittings seen in $\mathbf{8}$ are most likely due to the metal-metal interactions. 


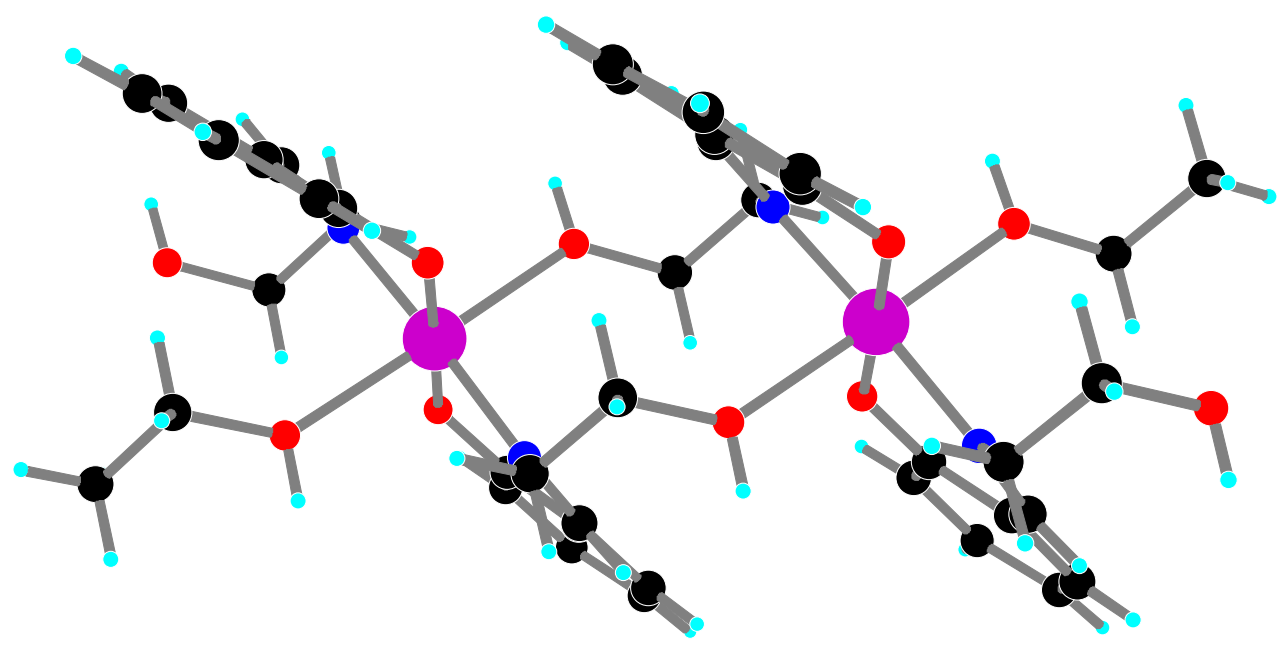

Figure S5. The arrangement of Mn moieties in 4 used to calculate the exchange integral $J$ in the polymer chain. $\mathrm{J}=0.02 \mathrm{~cm}^{-1}$ (antiferromagnetic, for $\mathrm{H}=\mathrm{JS}_{1} \mathrm{~S}_{2}$ ) was obtained.. The moieties are related by the symmetry operation $\mathrm{x}+1, \mathrm{y}, \mathrm{z}$ (a translation).

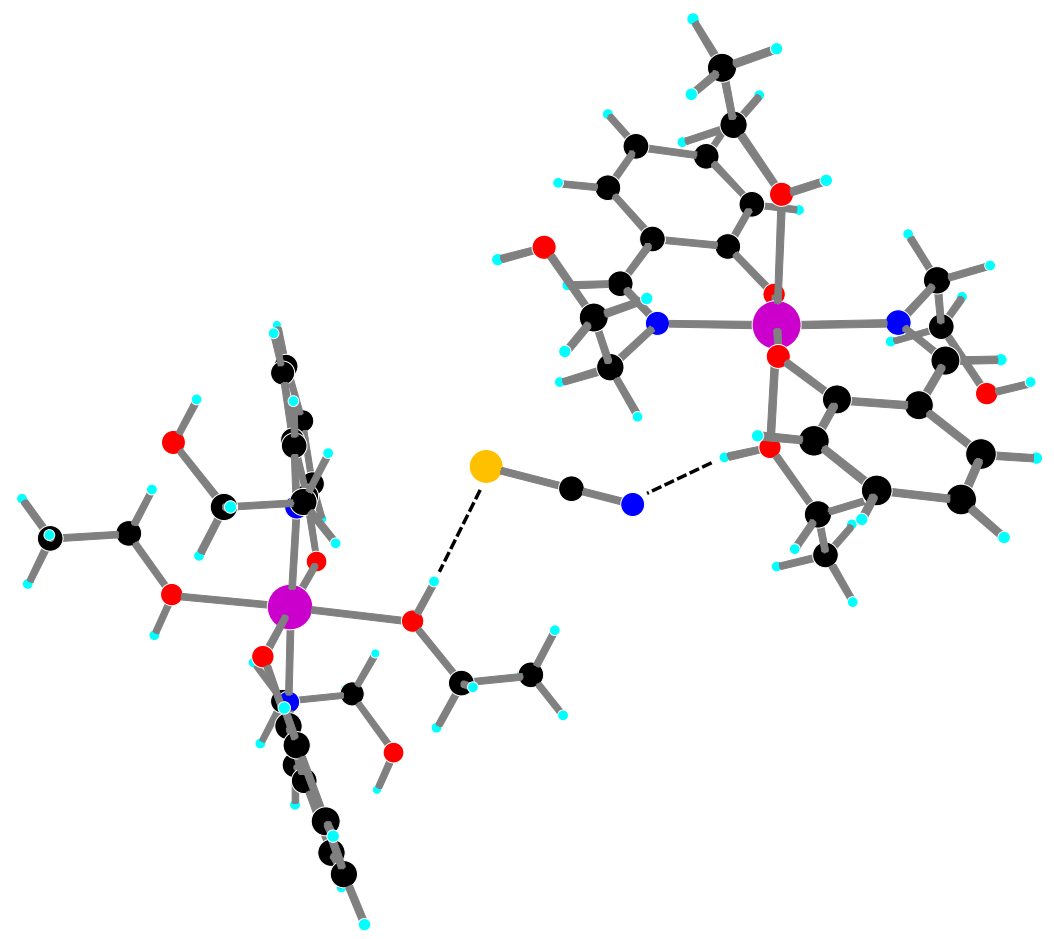

Figure S6. The arrangement of Mn moieties in 4, used to calculate the exchange integral $J$ due to the hydrogen bonds. $\mathrm{J}=0.02 \mathrm{~cm}^{-1}$ (antiferromagnetic) was obtained. The moieties are related by the symmetry operation $\mathrm{x}+3 / 2,-\mathrm{y}+1, \mathrm{z}-1 / 2$ (a 2 -fold axis) and they belong to different chains. 


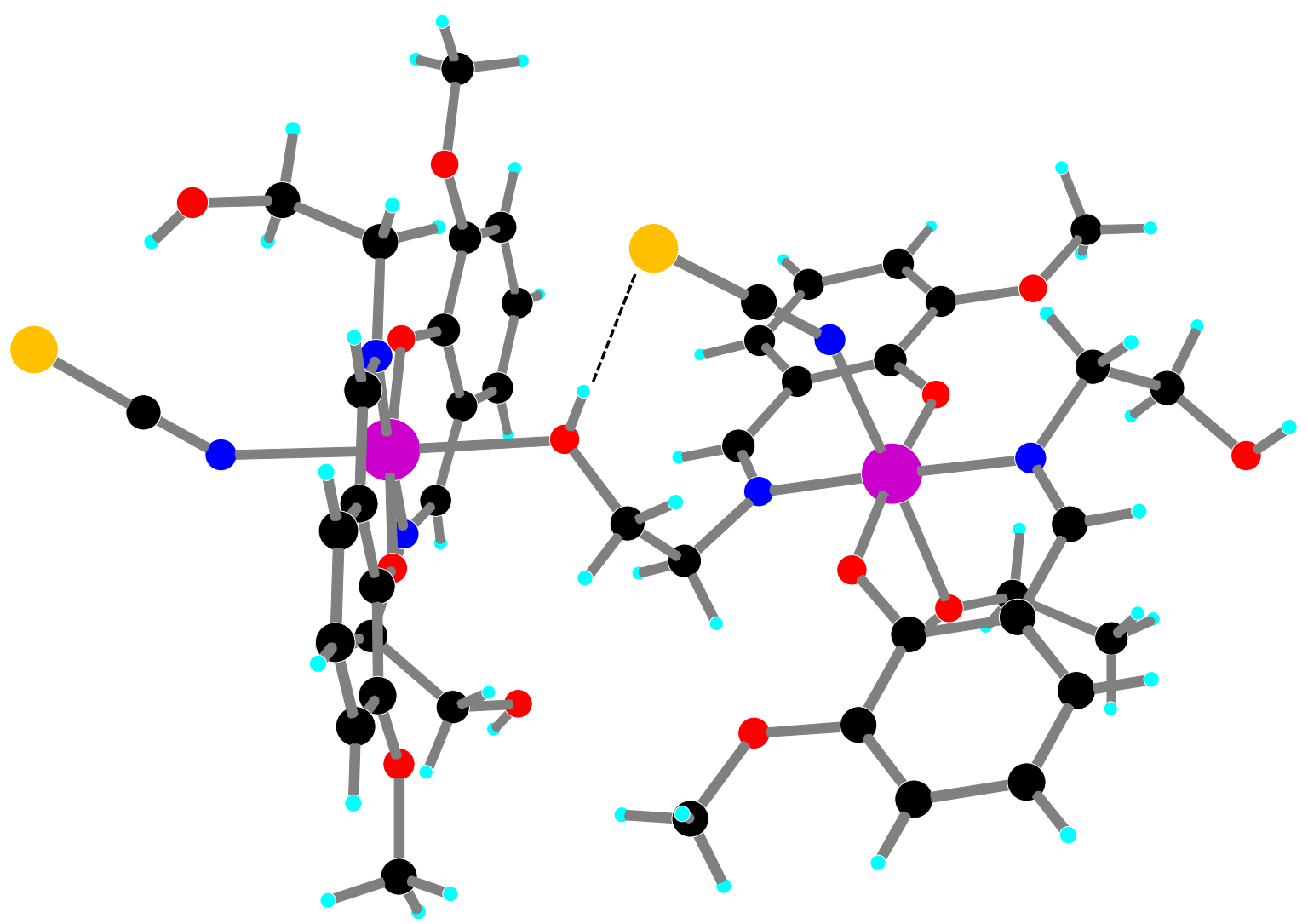

Figure S7. The arrangement of Mn moieties in $\mathbf{8}$ used to calculate the exchange integral $\mathrm{J}$ in the polymer chain. $\mathrm{J}=0.04 \mathrm{~cm}^{-1}$ (antiferromagnetic) was obtained. Note that the Mn moieties in the polymer chain of $\mathbf{8}$ are not parallel to each other, opposite to all other complexes. The two moieties are related by the symmetry operation $-x+3 / 2, y-$ $1 / 2,-\mathrm{z}+3 / 2$, (a 2-fold axis). 